Chirurg 2015 · 86:517-518

DOI 10.1007/s00104-015-0026-8

Online publiziert: 6. Juni 2015

(c) Springer-Verlag Berlin Heidelberg 2015

\section{Christiane J. Bruns}

Klinik für Allgemein-, Viszeral- und Gefäßchirurgie, Universitätsklinik Magdeburg, Magdeburg, Deutschland

\section{Komplikationen in der hepatopankreatobiliären Chirurgie}

\author{
Die Komplexität erfordert enge \\ interdisziplinäre Zusammenarbeit
}

Die chirurgische Therapie von Erkrankungen aus dem hepatopankreatobiliären Formenkreis erfordert komplexe operative Prozeduren. Morbidität (36-50\%) und Mortalität $(<5 \%)$ dieser Eingriffe konnten in den letzten Dekaden gesenkt werden. Insgesamt treten postoperative Komplikationen allerdings nach wie vor in bis zu 41 \% der Fälle auf, wobei die Pankreaschirurgie gegenüber hepatobilären Eingriffen mit einer deutlich höheren Komplikationsrate vergesellschaftet ist.

Das Auftreten einer postoperativen Pankreasfistel (POPF) ist eine der wichtigsten und potenziell lebensbedrohlichen Komplikationen nach Pankreasresektionen. Die Häufigkeit dieser Komplikation variiert zwischen $3 \%$ nach Pankreaskopfresektionen und bis zu 35\% nach distaler Pankreatektomie entsprechend der DISPACT-Studie. POPF können nach partieller Pankreatikoduodenektomie (PD) an der Pankreasanastomose auftreten, während sie nach distaler Pankreatektomie (DP) und Enukleationen am parenchymatösen Absetzungsrand und/oder am Pankreasgang lokalisiert sind. Die zentrale Pankreasteilresektion weist die höchste Fistelrate auf, da POPF sowohl an der Absetzungsfläche zum Pankreaskopf als auch an der Anastomose zum Pankreasschwanz hin auftreten können. Postoperativen Pankreasfisteln werden entsprechend der seit 2005 existierenden Definition durch die International Study Group of Pancreatic Surgery (ISGPS) abstufend in die Schweregrade von A bis C eingeteilt. Unkomplizierte
POPF (Grad A) können durch verlängerte Drainagedauer ohne weitere Maßnahmen austherapiert werden. Bei komplizierten POPF muss neben der Sicherstellung des adäquaten Ablaufs des Sekrets, bevorzugt durch interventionelle Drainage, die Therapie der POPF-assoziierten Komplikationen (Blutungen) erfolgen. Im Management sollten dabei immer alle Maßnahmen zur Vermeidung einer Reoperation ausgeschöpft werden, da eine Reoperation mit eventueller Restpankreatektomie eine erhebliche Morbidität und Mortalität aufweist, letztendlich allerdings immer als Salvage-Verfahren erwogen werden muss (Hackert T et al.).

\section{》) Zu den schwerwiegendsten Komplikationen zählen postoperative Blutungen}

Zu den schwerwiegendsten Komplikationen in der hepatopankreatobiliären Chirurgie zählen postoperative Blutungen, die mit einer Inzidenz von $6,7 \%$ nach Pankreaseingriffen und 3,2\% nach hepatobiliären Operationen auftreten. Frühe postoperative Blutungen sind auf operative Probleme zurückzuführen, späte Blutungen sind Ausdruck von Anastomoseninsuffizienzen, Fisteln oder Abszessen. Diese führen zur Gefäßarrosion oder der Entwicklung von Pseudoaneurysmata, meist mit vorausgehender, selbstlimitierender Sentinel-Blutung. Die jeweilige Therapie orientiert sich an dem Blutungszeitpunkt, der Lokalisation und dem Schweregrad der Blutung. Frühe postoperative Blutungen erfordern eine chirurgische Therapie, während bei späten Blutungen interventionelle Methoden die führenden Behandlungsverfahren sind, ausgenommen bei kreislaufinstabilen oder septischen $\mathrm{Pa}$ tienten. Essenziell ist zusätzlich zur Blutstillung - wenn möglich - die operative oder interventionelle Sanierung der zur Blutung führenden Ursache.

Neben Blutungen sind arterielle und portalvenöse Stenosen und Thrombosen ein Faktor für die postoperative Morbidität und Mortalität in der hepatopankreatobiliären Chirurgie. Thrombosen der Portalvene treten in ca. $9 \%$ nach Leberresektionen und $11 \%$ nach Pankreasresektionen mit Gefäßbeteiligung auf. Je nach Zeitpunkt der Diagnose und Symptomatik kommen operative, interventionelle oder konservative Verfahren zur Anwendung (Arend et al.).

Die häufigsten Spätkomplikationen/folgen nach Operationen an der Bauchspeicheldrüse sind exokrine Insuffizienz mit Malabsorptionssyndrom (80\%) und/ oder Diarrhö (30\%), endokrine Insuffizienz mit Diabetes mellitus (60\%), gastrointestinale Anastomosenulzera (6-18\%), Gallengangsstenosen (2-4\%), Pfortaderverschlüsse (20-30\%) und Schmerzen (20\%). Darüber hinaus können (peri-) pankreatische Pseudozysten und Flüssigkeitsretentionen als Folge kleiner Pankreassekretleckagen nach operativen oder interventionellen Eingriffen bzw. nach Pankreatitis oder Trauma auftreten. Die exokrine Pankreasinsuffizienz führt zum 
Malabsorptionssyndrom mit Verdauungsbeschwerden. Therapie der Wahl ist die orale Substitution von Pankreatin. Die endokrine Pankreasinsuffizienz führt zum pankreopriven Diabetes mellitus, wobei die hypoglykämische Krise besonders problematisch ist. Pseudozysten entwickeln sich aus Parenchymschäden, meist nach Pankreatitis, gelegentlich nach Pankreastrauma und selten als Operationsfolge. Die meisten Pseudozysten können endoskopisch zum Magen oder Duodenum drainiert werden. Komplexere Befunde erfordern allerdings ein offenchirurgisches Vorgehen mit Zystojejunostomie. Nach resezierenden Pankreaskopfeingriffen besteht ein erhöhtes gastrointestinales Ulkusrisiko, welches eine lebenslange postoperative Einnahme von Protonenpumpeninhibitoren indiziert.

Weitere Spätkomplikationen/-folgen mit limitierten Therapieoptionen sind Gallengangsstenosen (2-4\%) als Operationsfolge; Pfortaderthrombosen (30\%) und chronische Schmerzen (20\%) sind spätpostoperativ häufig durch die Grunderkrankung verursacht (Nitsche $U$ et al.).

\section{》) Dysregulierte \\ Funktionszustände der \\ Darmmotilität erhöhen \\ die Morbidität}

$\mathrm{Zu}$ den weiteren Komplikationen nach hepatopankreatobiliären Operationen mit deutlichem Anstieg der Morbidität zählen dysregulierte Funktionszustände der Darmmotilität sowie Entzündungen und Infektionen im Bereich der operierten Organe. Von besonderer Bedeutung ist, die primär funktionellen Störungen von sekundären Störungen der gastrointestinalen Mobilität zu unterscheiden, wobei letzteren als Symptom anderer postoperativer Komplikationen eine Bedeutung als Alarmsignal zukommt. Dazu zählt in erster Linie die verzögerte Magenentleerung (vME). Bei manifester vME gehören die Magenentlastung sowie die enterale oder parenterale Ernährung zum therapeutischen Standard.

Das Management der postoperativen Cholangitis und Pankreatitis beruht auf einer supportiven und antibiotischen Therapie. Bei der Wiederherstellung des
Galleabflusses bei Cholangitiden und der Drainage von Verhalten und Nekrosen kommen in zunehmendem Maße interventionelle und minimal-invasive Verfahren zur Anwendung (Schulze T et al.).

Auch rein biliäre Komplikationen nach hepatopankreatobiliären Eingriffen können schwerwiegende Folgen für die langfristige Lebensqualität der Patienten haben. Die zwei häufigsten Probleme hierbei sind zum einen die biliäre Stauung und zum anderen die Galleleckage oder Gallefistel. Beide Komplikationsformen gehen mit einer erheblichen Morbidität des Patienten einher und können somit den Krankenhausaufenthalt deutlich verlängern. Die adäquate und zeitgerechte Diagnose des vorliegenden Problems ist entscheidend. Sonographie, Computertomographie, Kontrastdarstellungen von Drainagen und endoskopisch retrograde Cholangiopankreatikographie (ERCP) sind die wegweisenden, bildgebenden Verfahren. Die frühzeitige operative Revision ist das erste Mittel im direkt postoperativen Verlauf. Interventionelle Verfahren wie ERCP und perkutane transhepatische Cholangiodrainage (+Stents und Drainagen) bieten eine Vielzahl weiterer Therapieoptionen (Dahlke $M$ et al.).

Biliäre Komplikationen nach Pankreasresektionen sind seltener, da die Gallengänge in vielen Fällen dilatiert und damit operativ technisch leichter zu versorgen sind. Zur einheitlichen Definition und zur Festlegung des Schweregrads einer biliären Leckage kann die Klassifikation der International Study Group of Liver Surgery (ISGLS) benutzt werden.

Die Komplexität der hepatopankreatobiliären Eingriffe erfordert enge interdisziplinäre Zusammenarbeit. Maßgeschneidertes Komplikationsmanagement trägt dazu bei, dass in Zukunft das Morbiditäts- und Mortalitätsrisiko nach hepatopankreatobiliären Eingriffen gesenkt werden kann.

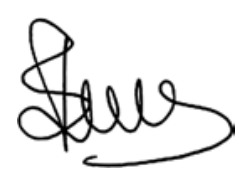

Prof. Dr. Christiane J. Bruns

\section{Korrespondenzadresse}

Prof. Dr. C.J. Bruns

Klinik für Allgemein-, Viszeral- und Gefäßchirurgie Universitätsklinik Magdeburg Leipziger Straße 44, 39120 Magdeburg

christiane.bruns@med.ovgu.de 